\title{
MDM4 expression in fibrolamellar hepatocellular carcinoma
}

\author{
ANJU KARKI ${ }^{1}$, JUAN PUTRA ${ }^{2}$, STEPHANIE S. KIM ${ }^{1}$, MICHAEL J. LAQUAGLIA ${ }^{1}$, \\ ANTONIO R. PEREZ-ATAYDE ${ }^{2}$, GHAZALEH SADRI-VAKILI ${ }^{3}$ and KHASHAYAR VAKILI ${ }^{1}$
}

Departments of ${ }^{1}$ Surgery and ${ }^{2}$ Pathology, Boston Children's Hospital, Harvard Medical School, Boston, MA 02115;
${ }^{3}$ Department of Neurology, Massachusetts General Hospital, Harvard Medical School, Boston, MA 02114, USA

Received January 28, 2019; Accepted June 25, 2019

DOI: $10.3892 /$ or.2019.7241

\begin{abstract}
Fibrolamellar hepatocellular carcinoma (FL-HCC) is a variant of hepatocellular carcinoma (HCC) that most commonly affects adolescents and young adults and is associated with an extremely poor prognosis due to the lack of effective chemotherapeutic agents. Mutations in p53 are a common oncogenic driver in $\mathrm{HCC}$ but not in FL-HCC. However, in tumors lacking a p53 mutation, the tumor suppressor activity of p53 has been revealed to be dysregulated in several different cancer types. One mechanism has been attributed to the overexpression of mouse double minute 4 protein (MDM4), a negative regulator of $\mathrm{p} 53$, which inhibits the normal functions of p53 including induction of apoptosis and DNA repair. Therefore, restoring the normal function of p53 in cancer cells by targeting MDM4 has become a potential therapeutic strategy. Hence, in the present study the components of the DNA damage response (DDR) pathway were examined; ATM, p53, and MDM4 in FL-HCC. Seven FL-HCC tumors along with their adjacent non-neoplastic hepatic tissues were examined. Ataxia-telangiectasia mutated (ATM), p53, and MDM4 protein expression was assessed using western blot analysis and cellular localization was determined using immunohistochemistry (IHC). MDM4 mRNA transcript levels were assessed using RT-qPCR. The present results demonstrated that the DNA damage sensor, ATM, is phosphorylated and localized to the nuclei of tumor cells. While there was a significant increase in total p53 protein in tumor cells, phosphorylated p53 was revealed to preferably localize to the cytoplasmic compartment of tumor cells. Notably, the present results revealed that MDM4 transcript levels were increased in the majority of tumor samples and the nuclear MDM4 levels were significantly increased in tumor tissue compared to their adjacent non-neoplastic liver tissue. The present results indicated that increased MDM4 expression
\end{abstract}

Correspondence to: Dr Khashayar Vakili, Department of Surgery, Boston Children's Hospital, Harvard Medical School, 300 Longwood Avenue, Boston, MA 02115, USA

E-mail: khashayar.vakili@childrens.harvard.edu

Key words: fibrolamellar hepatocellular carcinoma, p53, ATM, MDM4, DNA damage response and nuclear localization may be a potential mechanism for $\mathrm{p} 53$ dysregulation in FL-HCC.

\section{Introduction}

Hepatocellular carcinoma (HCC) is the third leading cause of cancer-related deaths worldwide (1). Fibrolamller hepatocellular carcinoma (FL-HCC) is a rare subtype of $\mathrm{HCC}$, which typically occurs in adolescents and young adults (2-4). FL-HCC has unique histological characteristics such as large, polygonal tumor cells with eosinophilic or oncocytic cytoplasm, large nuclei with prominent single nucleolus, and pericellular lamellar collagen fibrosis $(5,6)$ that sets it apart from conventional HCC. In addition, patients with FL-HCC generally do not have underlying liver disease (7) as opposed to conventional HCC which is commonly associated with cirrhosis and/or chronic viral hepatitis (8). Surgical resection is the predominant mode of treatment for FL-HCC and provides the only chance at a cure (9). However, the mortality rates remain high as a significant percentage of patients present with advanced or unresectable disease. Even patients who undergo surgical resection have a high risk of tumor recurrence. Therefore, overall survival of patients with FL-HCC will continue to be poor until effective chemotherapeutic agents are identified.

A recent approach to treating cancers has focused on activating the tumor suppressor function of p53, known as the 'guardian of the genome' $(10,11)$, which under normal conditions undergoes activation once there is DNA damage or instability (12). Ataxia-telangiectasia mutated (ATM), is a serine/threonine kinase that is often initially activated upon DNA damage $(13,14)$ and subsequently signals p53 to perform DNA repair, inhibit abnormal cell growth, or induce apoptosis (15-18). Conversely, p53 activity may be negatively regulated by mouse double minute 2 homolog (MDM2) or mouse double minute 4, human homolog (MDM4) (19). MDM2, an E3 ubiquitin-protein ligase, leads to the degradation of p53 through the proteasomal pathway and MDM4 exerts its inhibitory activity via binding to p53 transactivation domain (20-23).

Approximately $50 \%$ of all human cancers contain p53 mutations, which in turn results in the loss of p53 tumor suppressor function leading to cancer initiation and progression (24). There is now increasing evidence that even in cancers with non-mutated p53, the function of p53 is altered such that it 
favors the biology of the cancer cell and prevents the apoptosis of the transformed cell. One mechanism responsible for the dysregulation of p53 function has been attributed to the inhibitory effect of MDM4 $(25,26)$. The overexpression of MDM4 has been reported in various cancer types with non-mutated p53 (27). For example, MDM4 expression is elevated in $65 \%$ of human melanomas and in an animal model with Nras oncogene activation; overexpression of MDM4 increased cancer promotion in melanocytes (28). Similarly, in vitro studies of breast cancer lacking p53 mutations demonstrated that MDM4 knockdown inhibited cell growth (29). Collectively, these studies indicated that overexpression of MDM4 may result in dysregulation of p53 function and inhibiton of MDM4 may provide a therapeutic approach for treating cancers that lack p53 mutations.

As opposed to conventional HCC, which is commonly associated with mutations in p53 $(8,30), \mathrm{FL}-\mathrm{HCC}$ is not linked to any p53 mutations $(24,31,32)$, however, there is evidence for p53 pathway dysregulation based on transcriptome analysis of FL-HCC (33). Additionally, studies have reported that conventional HCC tumors lacking p53 mutations have increased MDM4 expression indicating that p53 function is disrupted $(1,31,34,35)$. Thus, in the present study, it was examined whether this tumor type is also associated with p53 dysregulation by examining the DDR components; ATM, p53, and MDM4.

\section{Materials and methods}

Tumor samples. Seven FL-HCC tumor samples and their matched non-neoplastic liver samples were obtained from patients who underwent hepatic resection or liver transplantation under an approved protocol by the Institutional Review Board (IRB) at Boston Children's Hospital. Written informed consent was obtained from the parents of patients under 18 years of age. The neoplastic and non-neoplastic tissues were reviewed by an expert liver pathologist (ARPA).

Histology and immunohistochemistry. Samples were fixed, embedded in paraffin, sectioned, and stained using conventional histological techniques by the Core Histology Facility at Boston Children's Hospital. Hematoxylin and eosin (H\&E) staining was performed on $4-\mu \mathrm{m}$ tissue sections as previously reported by LaQuaglia et al (36). Briefly, the paraffin slides were dehydrated for $60 \mathrm{sec}$ in methanol, followed by $120 \mathrm{sec}$ staining in Harris' Hematox ylin (Sigma Aldrich; Merck KGaA). The slides were washed in water for $20 \mathrm{sec}$. Next, the slides were incubated in the bluing reagent for $60 \mathrm{sec}$ (Thermo Fisher Scientific, Inc.) followed by 30 -sec washes in water and 95\% ethanol. The slides were further incubated in Eosin Yellow (Thermo Fisher Scientific, Inc.) for $60 \mathrm{sec}$ followed by a dehydration process in a series of ethanol and xylene. In order to perform immunohistochemistry (IHC), tissues were deparaffinized and retrieved with antigen unmasking solution (Vector Laboratories, Inc.). The sections were incubated in hydrogen peroxide (3\%) for 5 min for blocking the endogenous peroxidase activity. The samples were then blocked for $2 \mathrm{~h}$ at room termperature (RT) using normal goat serum-based reagent. Tissue sections were incubated overnight at $4^{\circ} \mathrm{C}$ using the following primary antibodies: MDMX (cat. no. NBP1-28862; dilution 1:100; Novus Biologicals, LLC), p53 (cat. no. PAb1801, dilution 1:100, Abcam) p-p53-S15 (cat. n. 9284S, dilution 1:150; Cell Signaling Technologies, Inc.), ATM (dilution 1:100; cat. no. NB100-104; Novus Biologicals, LLC), p-ATM-Ser1981 (cat. no. sc-47739; dilution 1:50; Santa Cruz Biotechnology, Inc.). The tissue sections were incubated in prediluted biotinylated anti-mouse or anti-rabbit secondary antibodies (cat. no. PK-8200; Vector Laboratories) for $45 \mathrm{~min}$ at room temperature followed by $1-\mathrm{h}$ incubation in Vecta Stain R.T.U (Vector Laboratories, Inc.) at room temperature. Vector reagents and DAB (diamonobenzidine) peroxidase substrate (Vector Laboratories, Inc.) were applied to detect the antibody staining.

Immunoblots, quantification and statistics. Tumor and non-neoplastic tissue samples were dissociated and lysed in ice-cold RIPA buffer supplemented with protease inhibitors, phosphatase inhibitors, using a Tissue Tearor Homogenizer (Biospec Products, Inc). Whole cell protein lysates (15-30 $\mu \mathrm{g}$ ) containing Lamelli buffer plus $\beta$-mercaptoethanol, were heated at $95^{\circ} \mathrm{C}$ for $10 \mathrm{~min}$. The samples were then resolved in $4-20 \%$ precast Tris-glycine gels (Invitrogen; Thermo Fisher Scientific, Inc.) and transferred onto PVDF membranes using Trans-Blot Turbo Transfer System (both from Bio-Rad Laboratories, Inc.). Membranes were subjected to blocking for $2 \mathrm{~h}$ at room termperature in 5\% non-fat dry milk in tris-buffered saline and $0.1 \%$ Tween-20. Immunoblot membranes were incubated in primary antibodies at $4^{\circ} \mathrm{C}$ overnight against the following antibodies: MDM4 (Abgent, Inc.; cat. no. ALS13152, dilution-1:1,000), p53 (Abcam; cat. no. PAb1801, dilution 1:1,000), phospho p53-S15 (Cell Signaling Technologies, Inc., cat. no. 9284S; dilution 1:1,000), PKA (Santa Cruz Biotechnology, Inc., cat. no. sc-903; dilution 1:1,000), $\alpha$-tubulin (GeneTex, Inc.; cat. no. GTX628802; dilution 1:3,000), GAPDH (Cell Signaling Technologies, Inc., cat. no. 97166; dilution 1:3,000 and GenTex, Inc., cat. no. GTX627408; dilution 1:3,000) and $\beta$-actin (Cell Signaling Technologies, Inc.; cat. no. 4970S; dilution 1:3,000). This was followed by three 10 -min washes. Membranes were then incubated in horseradish peroxidase (HRP) conjugated secondary antibodies (Jackson ImmunoResearch Laboratories, INC, anti-mouse- cat. no. 205-005-108; dilution 1:5,000, anti-rabbit; cat. no. 111-035-144; dilution 1:5,000) at room temperature for $45 \mathrm{~min}$ followed by three washes with TBST. Immunoblots were visualized using an enhanced chemiluminescence (ECL) kit (Bio-Rad Laboratories, Inc. and Thermo Fisher Scientific, Inc.). We did not have ample tumor tissue from patient P6 to perform all of the western blot analyses.

Protein quantification was performed using ImageJ software version:1.48v (National Institutes of Health) (normalized to GAPDH or $\beta$-actin). GraphPad Prism 7 (GraphPad Software) was used to generate graphs and Mann Whitney $\mathrm{U}$ test was used to generate P-values.

RNA expression analysis. Total cellular RNA from tumor and non-neoplastic liver samples was extracted using the E.Z.N.A midi kit (Qiagen, Inc.). One microgram of RNA was subjected to reverse transcription using the iScript cDNA synthesis kit (Bio-Rad Laboratories, Inc.). Gene amplification was performed using SYBR Rox and an AB 7000 Real-Time PCR system. Thermocycling conditions used were: 37 cycles 


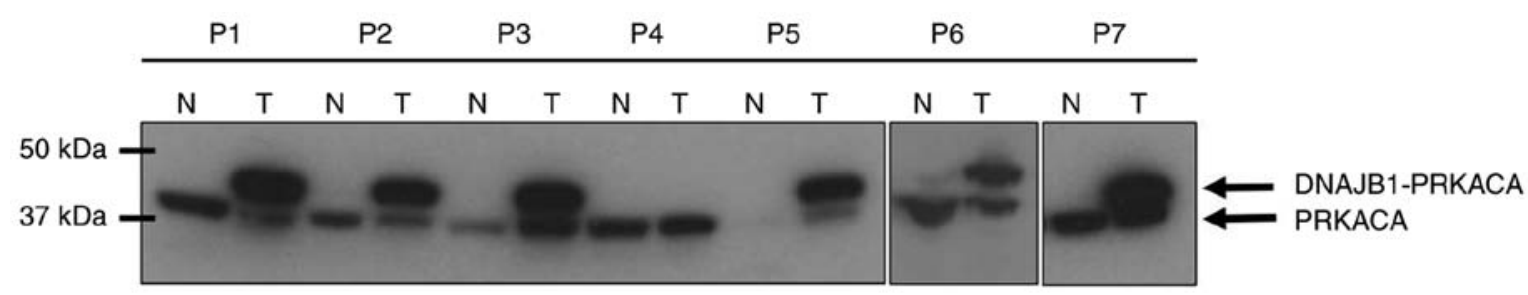

Figure 1. DNAJB1-PRKACA fusion protein in FL-HCC tumors. Western blot analysis using antibody against PRKACA protein. The native PRKACA band was observed at $\sim 41 \mathrm{kDa}$ and the presence of the fusion protein was demonstrated by a second band at $\sim 46 \mathrm{kDa}$. The $\mathrm{P} 4$ tumor was histologically characterized as FL-HCC but lacked the fusion protein. FL-HCC, fibrolamellar hepatocellular carcinoma; N, non-neoplastic; T, tumor.

at $95^{\circ} \mathrm{C}$ for $30 \mathrm{sec}, 59^{\circ} \mathrm{C}$ for $1 \mathrm{~min}$, and $72^{\circ} \mathrm{C}$ for $30 \mathrm{sec}$ with the following primers MDM4 (Hs00910358_s1) and GAPDH (Hs02758991_g1) (all from Applied Biosystems; Thermo Fisher Scientific, Inc.). Each gene reaction was performed in duplicates and all transcript values were normalized to $G A P D H$. The $\mathrm{Cq}$ values for each gene was generated using the AB7000 software and data analysis was performed using the $2^{-\triangle \Delta \mathrm{Cq}}$ method. The values were first normalized to $G A P D H$. Then to generate the final folds, the values were renormalized to the non-neoplastic samples.

Microscopy, semiquantative scoring system and statistical analysis. All images were obtained using an EVOS Imaging System (Invitrogen; Thermo Fisher Scientific, Inc.). In the present analysis for IHC, 5/7 tumor tissues were compared to non-neoplastic tissue from the same patient. The immunoreactive score (IRS) was utilized to evaluate each immunohistochemical stain and performed by a single pathologist (JP) (37). Histologic evaluation included the percentage of positive cells in the lesional and non-lesional areas and the staining intensity. IRS was a product of multiplication between the positive-cell proportion score (0-4) and staining intensity score (0-3). The final score was interpreted as negative (0-1), mild (2-3), moderate (4-8) or strongly positive (9-12). A detailed calculation for generating the IRS is illustrated in Table SI. Mann-Whitney test was performed to compare the median data values between non-neoplastic and neoplastic cells. The non-parametric two-sided Mann-Whitney test was conducted using Graphpad Prism 7 software for all statistical analyses and a P-value $<0.05$ was considered statistically significant.

\section{Results}

Patient characteristics and histopathology. All patients in the present study, were male and the median age of the patients in the present study was 13 years. Previous studies have reported that FL-HCC occurs predominantly in females (2), however, others have reported that both males and females are equally likely to be affected $(38,39)$. Two patients (P3 and P6) presented to our institution with metastatic disease. Patient P6 had prior liver resection at an outside institution and presented with recurrent metastatic disease and underwent tumor biopsy. The median follow-up for the entire cohort was 26 months (range 16-70 months). All patients except P7 had undergone neo-adjuvent therapy prior to surgery. Patient demographics, tumor data, and clinical course is summarized in Table I. Tumor and non-neoplastic livers from 4 patients were collected following hepatic resection; 2 after transplantation; and 1 following core-needle biopsy of a recurrent tumor. All tumors were reviewed by an expert liver pathologist (ARPA) and revealed to be characteristic of FL-HCC and all demonstrated microvascular invasion. All but one patient (P2) had tumor recurrence following surgery, at a median time of 155 days (range 0-563 days). Two patients succumbed to this disease since surgery, both due to tumor progression. Overall survival at 5 years was $71 \%$.

PRKACA-DNAJB1 fusion protein is expressed in the majority of $F L-H C C$. A recent study described the presence of PRKACA-DNAJBl fusion gene in chromosome 19 in FL-HCC tumors (40). This fusion gene results from a chromosomal deletion of 400 kilobase pairs, which generates a fusion chimera between exon 1 of DNAJB1 (Heat Shock Protein 40) and exons 2 through 10 of the catalytic subunit of protein kinase A, PRKACA $(40,41)$. Using western blot analysis, the presence of PRKACA-DNAJB1 fusion protein with an antibody targeting the c-terminus of PRKACA was assessed. It was revealed that $6 / 7$ tumor samples expressed the fusion protein (Fig. 1). The native PRKACA band was detected at $\sim 41 \mathrm{kDa}$ and the band shift at $\sim 46 \mathrm{kDa}$ in the immunoblots represented the presence of the fusion protein. Tumor sample from patient P4 was confirmed as FL-HCC histologically, however, the fusion protein was not present in this tumor.

p53 protein expression is increased in FL-HCC. The expression of p53 and its activated form, phosphorylated p53 (p-p53) in tumor tissue was then determined. Western blot analysis was performed from whole cell protein lysates using p53 and p-p53 (serine 15) antibodies and the corresponding protein levels were assessed. Quantitative analysis of the immunoblots revealed that p53 protein was significantly increased in tumor tissues compared to matching non-neoplastic liver tissues (Mann-Whitney test, $\mathrm{P}=0.03$, Fig. 2A and B). As revealed in Fig. 2C, individual immunoblot quantification demonstrated that p53 was increased in 5/6 tumor samples. Furthermore, western blot analysis revealed that although the overall p-p53 expression was not significantly elevated (Mann-Whitney test, $\mathrm{P}=0.39$ ), there was an increase in $\mathrm{p}-\mathrm{p} 53$ in $4 / 6$ tumors (Fig. 2D-F).

Next, the subcellular localization pattern of p53 and p-p53 was assessed. IHC was performed on paraffin-embedded sections using anti-p53 and anti-p-p53 antibodies followed by nuclear and cytoplasmic assessment of each protein using the IRS quantification system. There was very low nuclear and cytoplasmic p53 staining in both tumor and normal liver tissue (Fig. S1). In contrast, p-p53 was detected in both the 


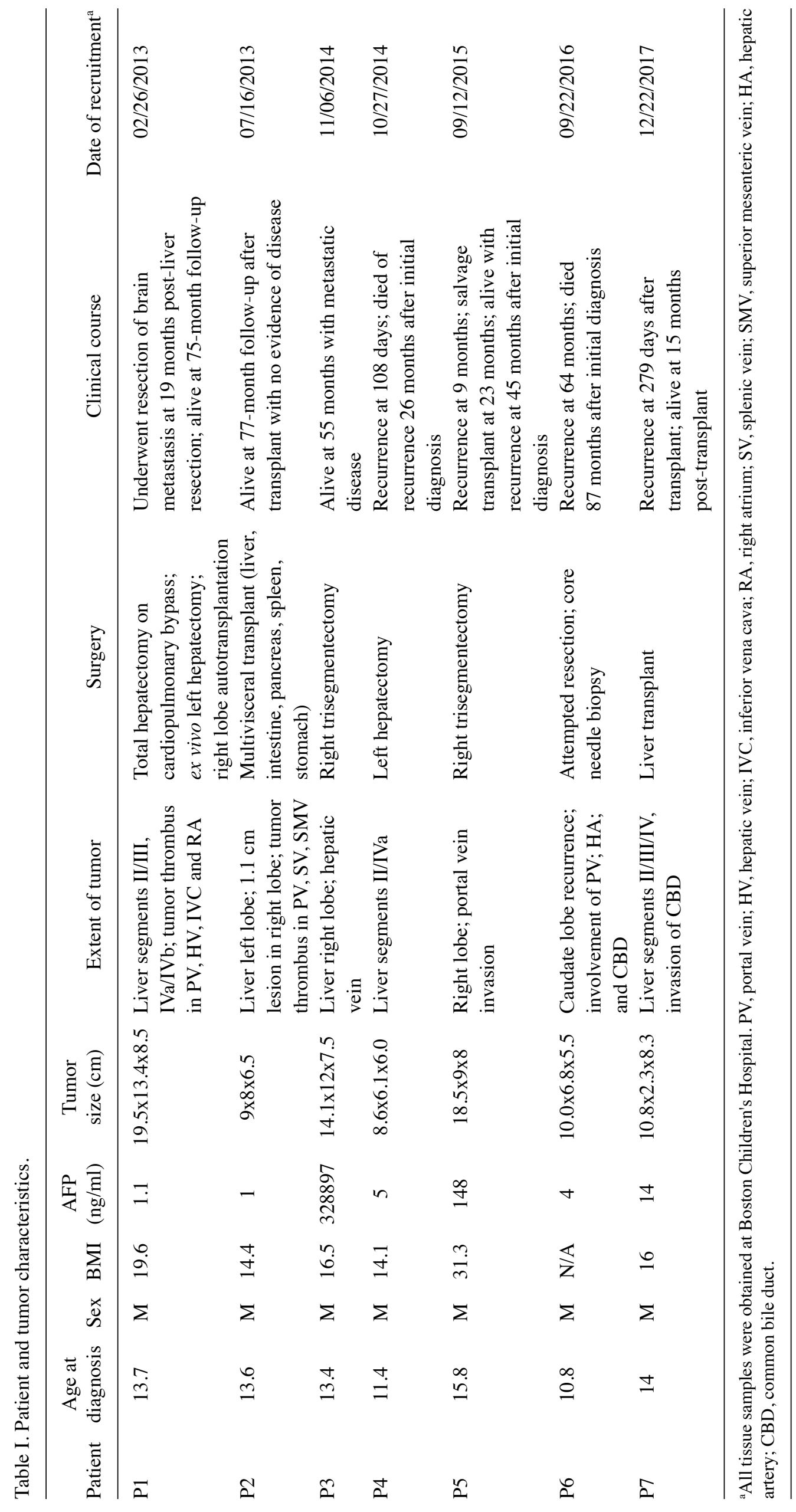


A

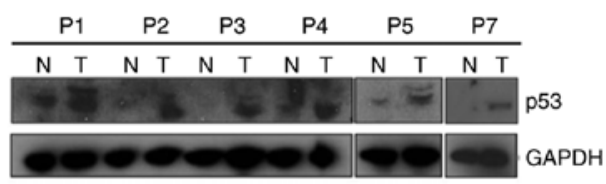

B

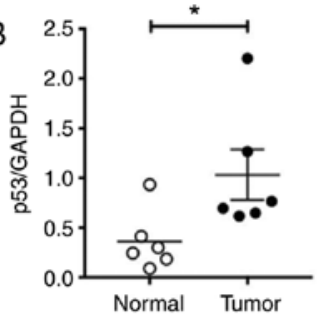

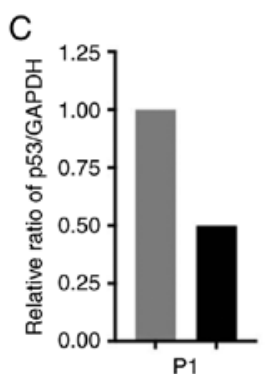

P1

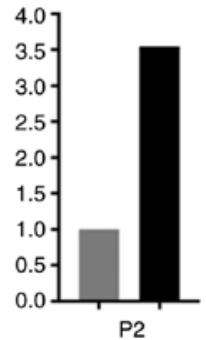

P2
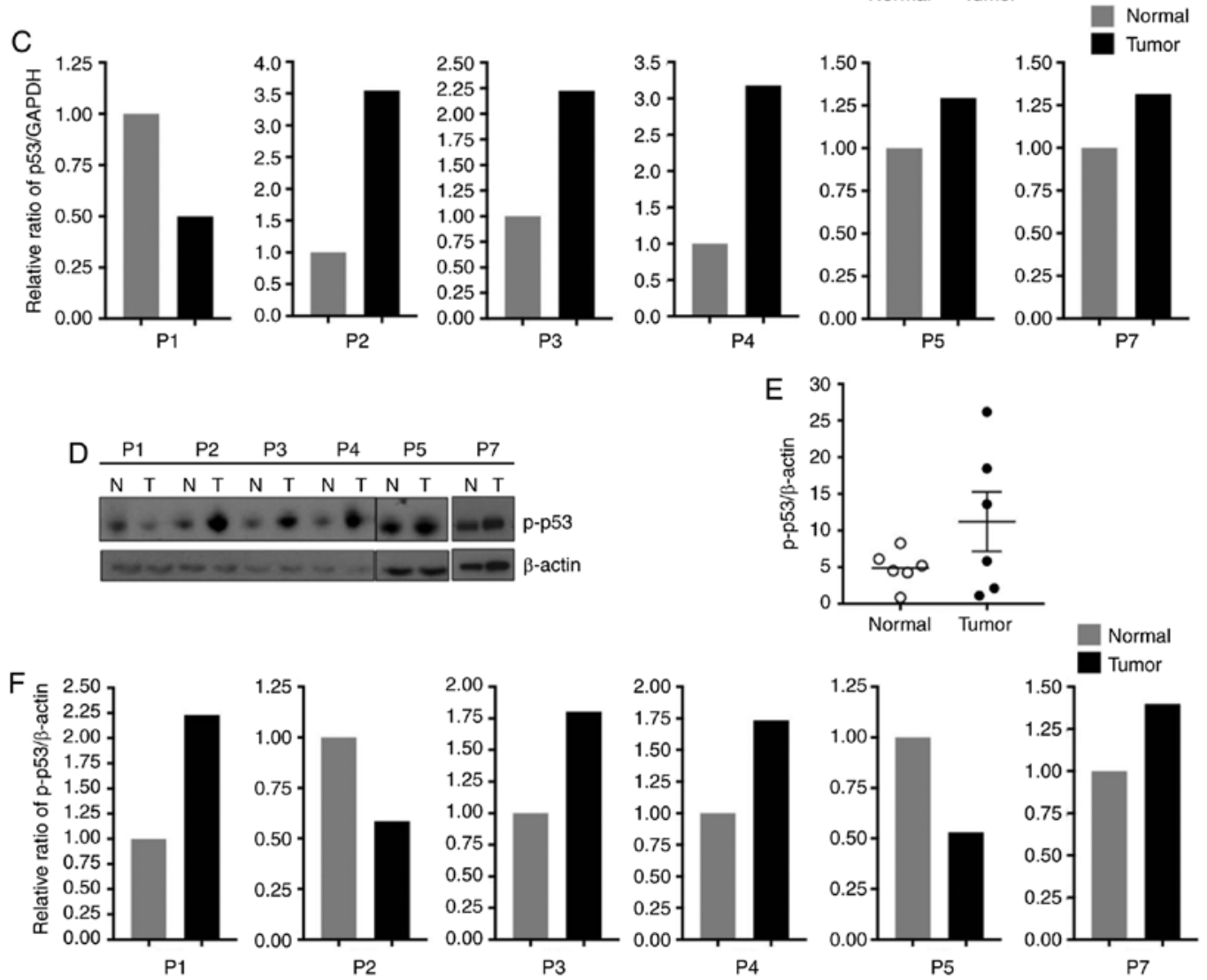

Figure 2. p53 protein expression is significantly upregulated in FL-HCC tumors. (A and B) Western blot analysis and densitometric quantification of p53 protein demonstrated higher p53 expression in tumors compared to non-neoplastic livers $\left({ }^{*} \mathrm{P}<0.05\right)$. (C) Densitometric analysis of p53 immunoblots demonstrating protein expression in individual patient samples. (D and E) Western blot analysis and densitometric quantification of FL-HCC tumors in comparison to the non-neoplastic liver. (F) Densitometric analysis of p-p53 immunoblots in individual patient samples. FL-HCC, fibrolamellar hepatocellular carcinoma; p-p53, phosphorylated p53; N, non-neoplastic; T, tumor.

nucleus and cytoplasm of tumor cells with no overall significant differences between tumor cells and non-neoplastic cells $(\mathrm{P}=0.28$ for nuclear $\mathrm{p}-\mathrm{p} 53$ and $\mathrm{P}=0.14$ for cytoplasmic $\mathrm{p}-\mathrm{p} 53$; Mann-Whitney test, Fig. 3A-C). However, the majority of the patients had higher IRS values for cytoplasmic p-p53 in the tumor cells compared to the non-neoplastic cells.

ATM, a p53 regulator, is activated in FL-HCC. Next, the expression and localization of ATM protein kinase, a known upstream regulator of $\mathrm{p} 53$, was examined (42). The detection of ATM and phosphorylated-ATM (serine 1981; p-ATM) protein levels was not feasible due to unsuitable antibodies for western blotting. However, protein expression and localization of ATM and p-ATM in tumors cells and non-neoplastic cells were examined using IHC (Fig. 3D and G) and IRS values were generated for nuclear and cytoplasmic localization of total ATM and p-ATM proteins (Fig. 3E and F and H and I, respectively). There was no statistically significant difference in nuclear or cytoplasmic ATM expression between tumor cells and non-neoplastic cells $(\mathrm{P}=0.20$ for nuclear ATM and $\mathrm{P}=0.16$ for cytoplasmic ATM; Mann-Whitney test). However, there was a significant increase in p-ATM levels in the nuclei of tumors compared to normal cells (Mann-Whitney test, $\mathrm{P}=0.005$, Fig. $3 \mathrm{H})$.

MDM4 transcipt levels are increased in the majority of FL-HCC tumors. Previous studies have reported that MDM4 inhibits the tumor suppressor activity of $\mathrm{p} 53$ by binding to its $\mathrm{N}$-terminus $(22,23)$ and increased expression of MDM4 has been reported in several other cancer types (25,28,34,35,43-45). The present quantitative RT-PCR results indicated that the relative transcript levels of MDM4 were increased in 4/7 tumors (Fig. 4A). However, when all samples were analyzed together, a statistically significant increase in $M D M 4$ transcript levels in FL-HCC tumors compared to their non-neoplastic liver tissue (Mann-Whitney test, $\mathrm{P}=0.69$, Fig. 4B) was not observed. This finding indicated that $M D M 4$ gene expression is increased in a proportion of FL-HCC tumors. 

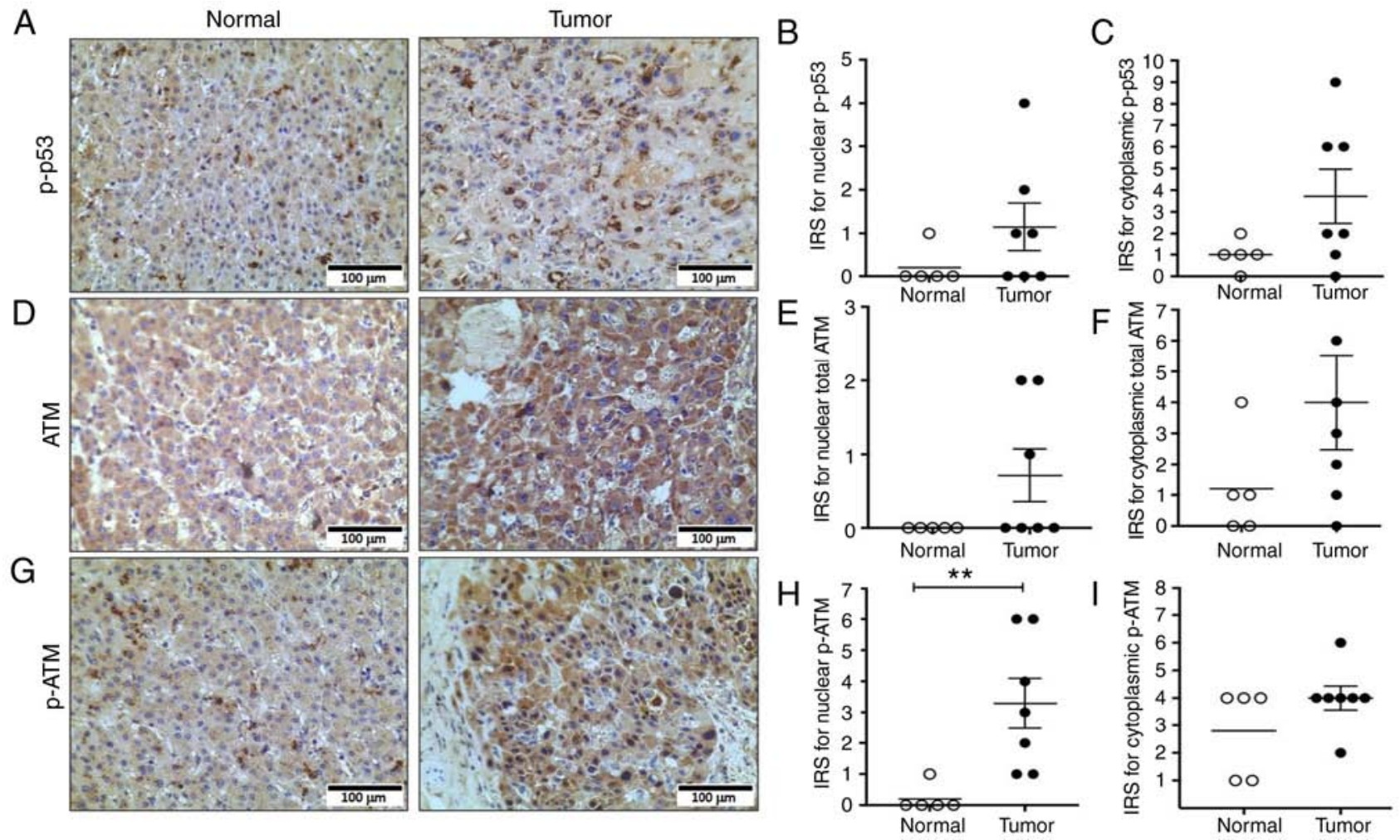

Figure 3. IHC of p53 and ATM in normal liver and tumor tissue. (A-C) Representative IHC for p-p53 and corresponding IRS for nuclear and cytoplasmic compartments. (D-F) Representative IHC of total ATM and corresponding IRS for nuclear and cytoplasmic compartments. (G-I) Representative IHC of $\mathrm{p}$-ATM and corresponding IRS for nuclear and cytoplasmic compartments demonstrating significant upregulation of nuclear $\mathrm{p}$-ATM in tumor cells $\left.{ }^{* *} \mathrm{P}<0.05\right)$. IHC, immunohistochemistry; ATM, ataxia-telangiectasia mutated; p-p53, phosphorylated p53; IRS, immunoreactive score; p-ATM, phosphorylated ATM.
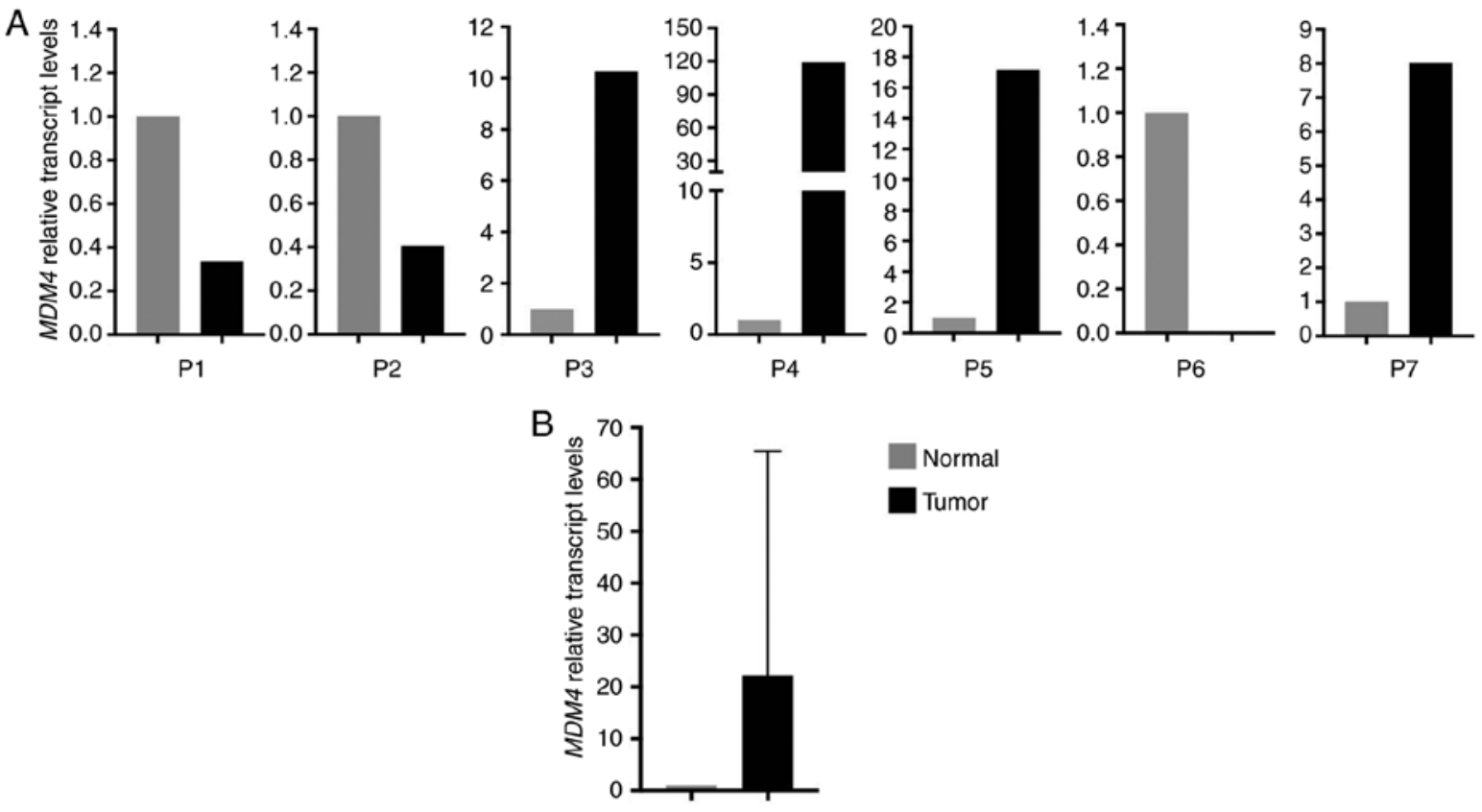

Figure 4. mRNA expression of MDM4 in FL-HCC tumors compared to non-neoplastic liver. (A) MDM4 transcript levels of individual tumor samples demonstrated a marked increase in 4/7 tumors. (B) Combined MDM4 transcript levels of all tumor samples. MDM4 transcript levels were normalized to GAPDH. $M D M 4$, mouse double minute 4; FL-HCC, fibrolamellar hepatocellular carcinoma.

MDM4 nuclear localization is significantly increased in FL-HCC. Given the increased MDM4 transcript levels in the majority of tumor samples, MDM4 protein expression was assessed. Western blot analysis revealed an increase in MDM4 protein levels in 4/6 tumor samples compared to the non-neoplastic liver (Fig. 5A and C) but it did not reach statistical significance when the samples were analyzed together (Mann-Whitney test, $\mathrm{P}=0.93$, Fig. 5B). However, there was a significant increase in nuclear 
A

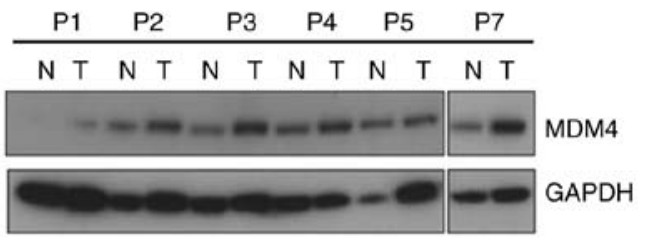

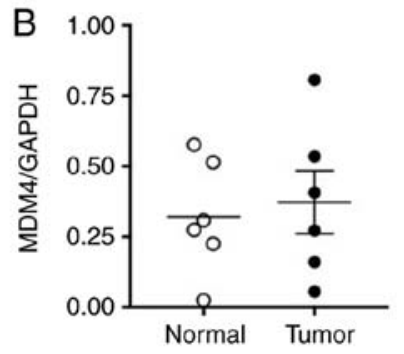
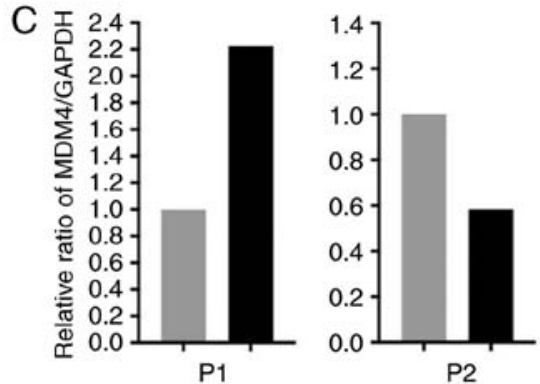

D

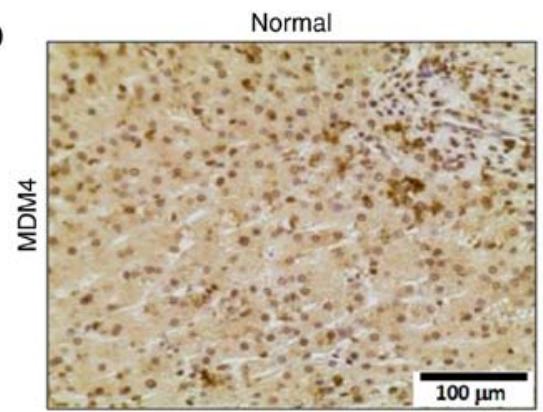

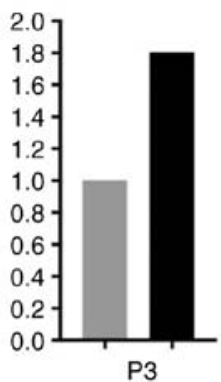
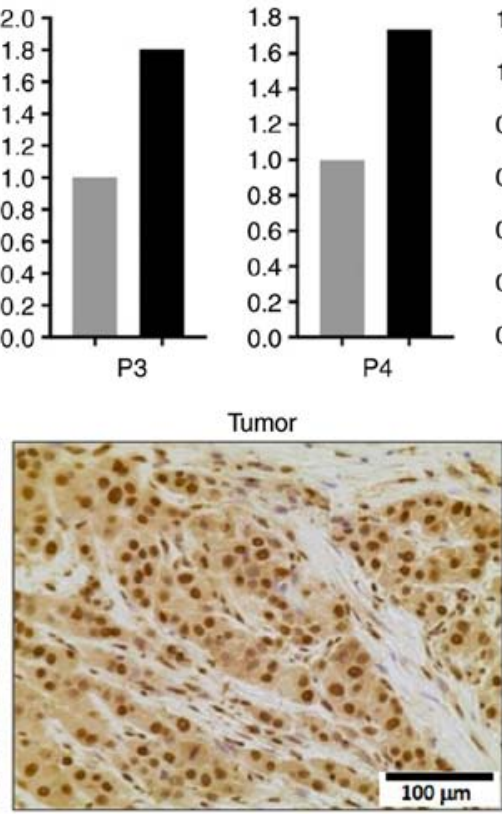
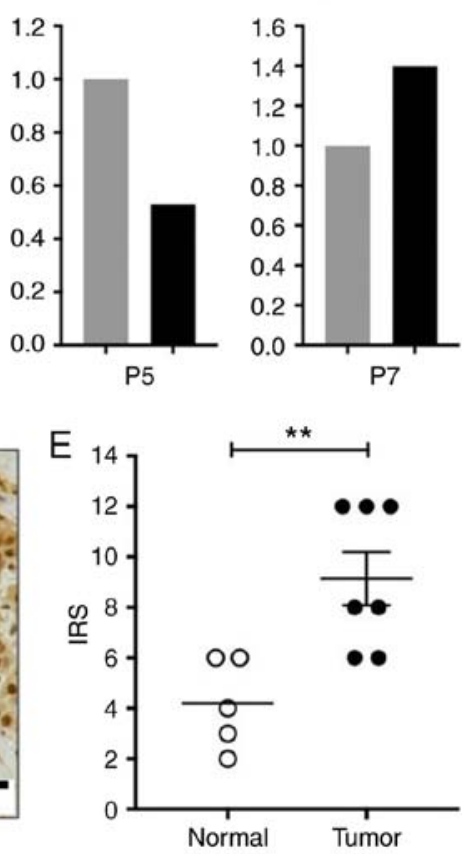

Figure 5. MDM4 protein expression and immunohistochemistry in FL-HCC tumors. (A-C) Western blot analysis and densitometric quantification of MDM4 protein expression in tumors and non-neoplastic livers. (D and E) Representative MDM4 IHC staining and IRS quantification demonstrating significant increase in MDM4 nuclear localization in tumor cells compared to non-neoplastic liver ( $\left.{ }^{* *} \mathrm{P}<0.01\right)$. MDM4, mouse double minute 4; FL-HCC, fibrolamellar hepatocellular carcinoma; IHC, immunohistochemistry; IRS, immunoreactive score.

MDM4 in tumor cells compared to non-neoplastic cells as revealed by IHC (Mann-Whitney test, $\mathrm{P}=0.009$; Fig. 5D and E).

\section{Discussion}

In the present study, the DDR pathway components p53, ATM, and MDM4 were examined in FL-HCC. The present findings indicated that the DDR pathway may be activated given the increase in nuclear p-ATM in FL-HCC. p53, the downstream target of ATM, was also increased in tumor tissues without any difference in its subcellular localization as assessed by IHC. In addition, the activated or phosphorylated form of p53 (p-p53) did not localize to the nucleus as predicted; instead p-p53 was localized to the cytoplasmic compartment in tumor samples. Moreover, the transcript levels of MDM4, a negative regulator of 553 , were increased in 4/7 tumors, however, protein nuclear localization was increased in all tumor samples (Table II).

FL-HCC was specifically focused on for the following reasons: i) FL-HCC does not commonly arise in a background of liver disease; ii) FL-HCC has a distinct histopathology compared to conventional HCC; and iii) unlike many conventional HCC types, FL-HCC does not contain multiple gene mutations, thereby representing a potentially homogeneous tumor type. A recent advance has been the revelation of a 400-kilobase pair deletion in chromosome 19 resulting in DNJAB1-PRKACA fusion transcript and protein in the majority of FL-HCC tumors $(40,41)$. A recent study using a mouse model demonstrated that expression of the fusion protein in the liver is sufficient to result in FL-HCC, thus demonstrating its role as an oncogenic driver (46). In the present study, the presence of this fusion protein was confirmed in $6 / 7$ of the tumor samples examined. Given that our cohort only had a single tumor that did not express the fusion protein, a conclusive determination was not feasible without a larger sample size. This highlights the major limitations of conducting cancer research on rare tumors such as FL-HCC which include: i) Small sample size; ii) limited amount of patient-derived tumor tissue; and iii) lack of in vitro or in vivo models.

p53 is one of the key components of the DDR pathway and its transcriptional activity has been revealed to prevent aberrant oncogenic cell growth. Studies have reported that p53 is mutated in $\sim 50 \%$ of lung, liver, breast, brain, and prostate cancers (24,47-49). However, mutations in p53 have not been commonly observed in FL-HCC (40,50). Upon detection of cellular stress and DNA damage, ATM is activated which subsequently activates 53 to induce downstream target genes to repair DNA damage, halt cell growth, or induce apoptosis (51-54). In fact, in the FL-HCC tumor tissue there was 
Table II. Summary of tumor IHC and MDM4 transcript levels.

\begin{tabular}{lccccccc}
\hline Patient & $\begin{array}{c}\text { DNAJB1-PRKACA } \\
\text { fusion present }\end{array}$ & $\begin{array}{c}\text { MDM4 } \\
\text { transcript levels }\end{array}$ & MDM4 & $\begin{array}{c}\text { Nuclear } \\
\text { p-ATM }\end{array}$ & $\begin{array}{c}\text { Cytoplasmic } \\
\text { p-ATM }\end{array}$ & $\begin{array}{c}\text { Nuclear } \\
\text { p-p53 }\end{array}$ & $\begin{array}{c}\text { Cytoplasmic } \\
\text { p-p53 }\end{array}$ \\
\hline P1 & Yes & 0.34 & +++ & + & ++ & - & +++ \\
P2 & Yes & 0.41 & ++ & - & ++ & - & + \\
P3 & Yes & 10.26 & +++ & + & ++ & ++ \\
P4 & No & 119.25 & +++ & ++ & ++ & ++ \\
P5 & Yes & 17.18 & ++ & ++ & + & + \\
P6 & Yes & 0.00006 & ++ & - & ++ & - & + \\
P7 & Yes & 8.01 & ++ & ++ & ++ & + \\
\hline
\end{tabular}

IHC IRS score: 0-1 (-); 2-3 (+); 4-8 (++); 9-12 (+++). IHC, immunohistochemistry; MDM4, mouse double minute 4; IRS, immunoreactive score; p-p53, phosphorylated p53; p-ATM, phosphorylated ATM.

increased nuclear localization of phosphorylated ATM (Fig. 3), indicating that the DNA damage repair process is activated in these tumors. Under normal conditions, activated p53 or phosphorylated p53 localizes to the nucleus where it performs its transcriptional and tumor suppressor activity. However, despite the increased phosphorylated ATM in the nucleus, the tumor samples assessed in the present study lacked nuclear p-p53 as demonstrated in Fig. 3A-C. In fact, phosphorylated p53 preferentially localized to the cytoplasm of FL-HCC tumor cells as opposed to the nucleus. These findings confirmed previously published studies, which demonstrated a loss of tumor suppressive function of non-mutant p53 via translocation to the cytoplasm in several cancers (55-57). Notably, cancers lacking nuclear p53 are not responsive to chemotherapy or radiotherapy similar to $\mathrm{FL}-\mathrm{HCC}(55,56,58,59)$.

In the present study, the tumors demonstrated activation of ATM which is an upstream regulator of p53. ATM activation may be due to i) tumor cell DNA instability and attempt at DNA repair or apoptosis or ii) a result of the toxic effects of neo-adjuvant chemotherapy (60). In either case, the expected response of p53 to elicit DNA damage repair or apoptosis was not observed. Clinically, it is clear that FL-HCC tumors are not responsive to conventional chemotherapy which was also evident in our cohort given the high rate of tumor progression and recurrence. On the other hand, assuming that ATM activation is a result of the cell's recognition of DNA instability following malignant transformation, the normal function of p53 is still compromised. In our tumor cohort a significant nuclear increase in the p53 negative regulator, MDM4, was observed. Therefore, it is possible that increased MDM4 results in inhibition of the normal function of p53 in these tumors. The exact mechanism for increased MDM4 expression and nuclear localization is unclear, although potential mechanisms may include increased gene expression; decreased protein degradation due to changes in post-transcriptional regulation; or alteration in post-translational regulation of MDM4. For example, recent studies have demonstrated that levels of miRNA-128 and miRNA-370 are decreased in pancreatic and colon cancer, respectively $(61,62)$. These studies demonstrated that the aforementioned miRNAs can directly inhibit MDM4 and promote cancer cell apoptosis. The role of miRNAs in the regulation of MDM4 in FL-HCC is unknown at this time and requires further research by examining the differential expression of known MDM4-specific miRNA in tumor cells compared to normal hepatocytes.

In the cancers which do not have mutated p53, there is evidence that tumor cells inhibit the normal function of $\mathrm{p} 53$ and prevent the cell from committing to DNA repair or apoptosis. Even in tumors with p53 mutation, studies have focused on re-establishing the normal function of p53 despite the presence of its mutated form. For example, in vitro studies have revealed that small molecules can reverse the aberrant function of mutant p53 and subsequently induce apoptosis (63-65). Therefore, the idea of reactivating or restoring the function of p53 for the treatment of cancer has been gaining interest $(12,66)$. One strategy to restore the normal function of non-mutated p53 is to target its negative regulators such as MDM4. MDM4 induces its inhibitory effect by binding to the N-terminus transactivation domain of p53 and affecting its transcriptional activity $(22,23,67)$. Recent studies have revealed that MDM4 is frequently overexpressed in a number of cancer types lacking p53 mutations including breast, melanoma, retinoblastoma, acute myeloid leukemia, and esophageal squamous cell carcinoma $(25,28,43-45,68,69)$. Therefore, in these cancers, inhibition of MDM4 activity has been an appealing therapeutic strategy to reactivate the function of p53 and ultimately control aberrant cell proliferation and tumor progression. In light of these findings, there is an interest in identifying small molecules or peptides that specifically target MDM4. In fact, MDM4 inhibitors have exhibited promising efficacy in both in vitro and in vivo models of cancers. Wang et al demonstrated that the MDM4 inhibitor, NSC207895, restored normal function of p53 in breast cancer cell lines and triggered cell apoptosis (70). Likewise, knockdown of MDM4 in breast cancer cell lines and xenograft models was revealed to induce senescence of cancer cells (29). In addition, in retinoblastoma cells, MDM4 inhibitor, SJ-172550, effectively triggered cell death (71). Lastly, in melanoma cells, known to be resistant to BRAF (B-Raf proto oncogene, serine threonine kinase) inhibitors, the inhibition of p53-MDM4 interaction using the MDM4 inhibitor, SAH-p53-8, exhibited effective activation of p53 and reduction in cell growth (28). Collectively, these studies indicated that inhibiting MDM4 can restore p53 activity in 
cancers lacking p53 mutations, thereby providing a promising new therapeutic strategy. To the best of our knowledge, the present study is the first to specifically examine MDM4 in FL-HCC. Whether p53 dysregulation is directly due to MDM4 activity in this tumor type is yet unclear, however, given the existing evidence in other cancers, it is a plausible hypothesis. In conclusion, our finding of increased nuclear MDM4 in FL-HCC tumors unveils a potential new target for the development of novel treatments for this highly lethal cancer.

\section{Acknowledgements}

We would like to thank Dr Amanda Dios, Kaly Mueller, Eric Granucci, Dr Ina Kycia, Dr Sanjeev Vasudevan and Dr Sarah Woodfield for their scientific advice on this study.

\section{Funding}

The present research was funded by CHMC Surgical Foundation, Inc.

\section{Author contributions}

AK and KV designed and AK performed all experiments. JP and ARPA reviewed the pathology slides and quantified the staining sections. AK, MJL, SSK, GSV and KV analyzed all data. AK and KV wrote the manuscript and all co-authors reviewed the final manuscript and agree to be accountable for all aspects of the research in ensuring that the accuracy or integrity of any part of the work are appropriately investigated and resolved.

\section{Availability of data and materials}

The datasets used and/or analyzed during the current study are available from the corresponding author on reasonable request.

\section{Ethics approval and consent to participate}

The use of human tissue in the present study was approved by the Institutional Review Board at Boston Children's Hospital. Patients and/or patient's guardians provided consent for the use of tissue in the present study.

\section{Patient consent for publication}

Not applicable.

\section{Competing interests}

The authors do not have any personal, professional, or financial relationships that would constitute a competing interest in regards to this work.

\section{References}

1. Schlaeger C, Longerich T, Schiller C, Bewerunge P, Mehrabi A, Toedt G, Kleeff J, Ehemann V, Eils R and Lichter P, et al: Etiology-dependent molecular mechanisms in human hepatocarcinogenesis. Hepatology 47: 511-520, 2008.
2. El-Serag HB and Davila JA: Is fibrolamellar carcinoma different from hepatocellular carcinoma? A US population-based study. Hepatology 39: 798-803, 2004.

3. Mavros MN, Mayo SC, Hyder O and Pawlik TM: A systematic review: Treatment and prognosis of patients with fibrolamellar hepatocellular carcinoma. J Am Coll Surg 215: 820-830, 2012.

4. Kakar S, Chen X, Ho C, Burgart LJ, Sahai V, Dachrut S, Yabes A, Jain D and Ferrell LD: Chromosomal changes in fibrolamellar hepatocellular carcinoma detected by array comparative genomic hybridization. Mod Pathol 22: 134-141, 2009.

5. Craig JR, Peters RL, Edmondson HA and Omata M: Fibrolamellar carcinoma of the liver: A tumor of adolescents and young adults with distinctive clinico-pathologic features. Cancer 46: 372-379, 1980.

6. Kakar S, Burgart LJ, Batts KP, Garcia J, Jain D and Ferrell LD: Clinicopathologic features and survival in fibrolamellar carcinoma: Comparison with conventional hepatocellular carcinoma with and without cirrhosis. Mod Pathol 18: 1417-1423, 2005.

7. Edmondson HA: Differential diagnosis of tumors and tumor-like lesions of liver in infancy and childhood. AMA J Dis Child 91: 168-186, 1956.

8. Sanyal AJ, Yoon SK and Lencioni R: The etiology of hepatocellular carcinoma and consequences for treatment. Oncologist 15 (Suppl 4): S14-S22, 2010

9. Lafaro KJ and Pawlik TM: Fibrolamellar hepatocellular carcinoma: Current clinical perspectives. J Hepatocell Carcinoma 2: 151-157, 2015.

10. Lane DP: Cancer. p53, guardian of the genome. Nature 358: 15-16, 1992.

11. Efeyan A and Serrano M: p53: Guardian of the genome and policeman of the oncogenes. Cell Cycle 6: 1006-1010, 2007.

12. Selivanova G: Wild type p53 reactivation: From lab bench to clinic. FEBS Lett 588: 2628-2638, 2014.

13. Shiloh Y: ATM: Ready, set, go. Cell Cycle 2: 116-117, 2003.

14. Shiloh Y: ATM and related protein kinases: Safeguarding genome integrity. Nat Rev Cancer 3: 155-168, 2003.

15. Banin S, Moyal L, Shieh S, Taya Y, Anderson CW, Chessa L, Smorodinsky NI, Prives C, Reiss Y, Shiloh Y and Ziv Y: Enhanced phosphorylation of p53 by ATM in response to DNA damage. Science 281: 1674-1677, 1998.

16. Polyak K, Xia Y, Zweier JL, Kinzler KW and Vogelstein B: A model for p53-induced apoptosis. Nature 389: 300-305, 1997.

17. Livingstone LR, White A, Sprouse J, Livanos E, Jacks T and Tlsty TD: Altered cell cycle arrest and gene amplification potential accompany loss of wild-type p53. Cell 70: 923-935, 1992.

18. Sengupta $S$ and Harris CC: p53: Traffic cop at the crossroads of DNA repair and recombination. Nat Rev Mol Cell Biol 6: 44-55, 2005.

19. Marine JC: MDM2 and MDMX in cancer and development. Curr Top Dev Biol 94: 45-75, 2011.

20. Marine JC and Jochemsen AG: Mdmx as an essential regulator of p53 activity. Biochem Biophys Res Commun 331: 750-760, 2005.

21. Haupt Y, Maya R, Kazaz A and Oren M: Mdm2 promotes the rapid degradation of p53. Nature 387: 296-299, 1997.

22. Shvarts A, Bazuine M, Dekker P, Ramos YF, Steegenga WT, Merckx G, van Ham RC, van der Houven van Oordt W, van der Eb AJ and Jochemsen AG: Isolation and identification of the human homolog of a new p53-binding protein, Mdmx. Genomics 43: 34-42, 1997.

23. Shvarts A, Steegenga WT, Riteco N, van Laar T, Dekker P, Bazuine M, van Ham RC, van der Houven van Oordt W, Hateboer G, van der Eb AJ and Jochemsen AG: MDMX: A novel p53-binding protein with some functional properties of MDM2. EMBO J 15: 5349-5357, 1996.

24. Leroy B, Anderson M and Soussi T: TP53 mutations in human cancer: Database reassessment and prospects for the next decade. Hum Mutat 35: 672-688, 2014.

25. Li L, Tan Y, Chen X, Xu Z, Yang S, Ren F, Guo H, Wang X, Chen Y, Li G and Wang H: MDM4 overexpressed in acute myeloid leukemia patients with complex karyotype and wild-type TP53. PLoS One 9: e113088, 2014.

26. Quintás-Cardama A, Hu C, Qutub A, Qiu YH, Zhang X, Post SM, Zhang N, Coombes K and Kornblau SM: p53 pathway dysfunction is highly prevalent in acute myeloid leukemia independent of TP53 mutational status. Leukemia 31: 1296-1305, 2017.

27. Li Q and Lozano G: Molecular pathways: Targeting Mdm2 and Mdm4 in cancer therapy. Clin Cancer Res 19: 34-41, 2013.

28. Gembarska A, Luciani F, Fedele C, Russell EA, Dewaele M, Villar S, Zwolinska A, Haupt S, de Lange J, Yip D, et al: MDM4 is a key therapeutic target in cutaneous melanoma. Nat Med 18: 1239-1247, 2012. 
29. Haupt S, Buckley D, Pang JM, Panimaya J, Paul PJ, Gamell C, Takano EA, Lee YY, Hiddingh S, Rogers TM, et al: Targeting Mdmx to treat breast cancers with wild-type p53. Cell Death Dis 6: e1821, 2015

30. Kan Z, Zheng H, Liu X, Li S, Barber TD, Gong Z, Gao H, Hao K, Willard MD, Xu J, et al: Whole-genome sequencing identifies recurrent mutations in hepatocellular carcinoma. Genome Res 23: 1422-1433, 2013.

31. Kunst C, Haderer M, Heckel S, Schlosser S and Müller M: The p53 family in hepatocellular carcinoma. Transl Cancer Res 5: 632-638, 2016

32. Ward SC and Waxman S: Fibrolamellar carcinoma: A review with focus on genetics and comparison to other malignant primary liver tumors. Semin Liver Dis 31: 61-70, 2011.

33. Sorenson EC, Khanin R, Bamboat ZM, Cavnar MJ, Kim TS, Sadot E, Zeng S, Greer JB, Seifert AM, Cohen NA, et al: Genome and transcriptome profiling of fibrolamellar hepatocellular carcinoma demonstrates p53 and IGF2BP1 dysregulation. PLoS One 12: e0176562, 2017.

34. Cancer Genome Atlas Research Network. Electronic address: wheeler@bcm.edu; Cancer Genome AtlasResearch Network: Comprehensive and integrative genomic characterization of hepatocellular carcinoma. Cell 169: 1327-1341.e23, 2017.

35. Arai Y, Honda S, Haruta M, Kasai F, Fujiwara Y, Ohshima J, Sasaki F, Nakagawara A, Horie H, Yamaoka H, et al Genome-wide analysis of allelic imbalances reveals $4 \mathrm{q}$ deletions as a poor prognostic factor and MDM4 amplification at 1q32.1 in hepatoblastoma. Genes Chromosomes Cancer 49: 596-609, 2010.

36. LaQuaglia MJ, Grijalva JL, Mueller KA, Perez-Atayde AR, Kim HB, Sadri-Vakili G and Vakili K: YAP subcellular localization and Hippo pathway transcriptome analysis in pediatric hepatocellular carcinoma. Sci Rep 6: 30238, 2016.

37. Fedchenko N and Reifenrath J: Different approaches for interpretation and reporting of immunohistochemistry analysis results in the bone tissue-a review. Diagn Pathol 9: 221, 2014.

38. Hemming AW, Langer B, Sheiner P, Greig PD and Taylor BR Aggressive surgical management of fibrolamellar hepatocellular carcinoma. J Gastrointest Surg 1: 342-346, 1997.

39. Stevens WR, Johnson CD, Stephens DH and Nagorney DM: Fibrolamellar hepatocellular carcinoma: Stage at presentation and results of aggressive surgical management. AJR Am J Roentgenol 164: 1153-1158, 1995.

40. Honeyman JN, Simon EP, Robine N, Chiaroni-Clarke R, Darcy DG, Lim, II, Gleason CE, Murphy JM, Rosenberg BR, Teegan L, et al: Detection of a recurrent DNAJB1-PRKACA chimeric transcript in fibrolamellar hepatocellular carcinoma. Science 343: 1010-1014, 2014.

41. Graham RP, Jin L, Knutson DL, Kloft-Nelson SM, Greipp PT, Waldburger N, Roessler S, Longerich T, Roberts LR, Oliveira AM, et al: DNAJB1-PRKACA is specific for fibrolamellar carcinoma. Mod Pathol 28: 822-829, 2015.

42. Turenne GA, Paul P, Laflair L and Price BD: Activation of $\mathrm{p} 53$ transcriptional activity requires ATM's kinase domain and multiple $\mathrm{N}$-terminal serine residues of p53. Oncogene 20: 5100-5110, 2001.

43. Haupt S, Vijayakumaran R, Miranda PJ, Burgess A, Lim E and Haupt Y: The role of MDM2 and MDM4 in breast cancer development and prevention. J Mol Cell Biol 9: 53-61, 2017.

44. Zhang H, Hu L, Qiu W, Deng T, Zhang Y, Bergholz J and Xiao ZX: MDMX exerts its oncogenic activity via suppression of retinoblastoma protein. Oncogene 34: 5560-5569, 2015.

45. Carneiro A, Isinger A, Karlsson A, Johansson J, Jönsson G, Bendahl PO, Falkenback D, Halvarsson B and Nilbert M: Prognostic impact of array-based genomic profiles in esophageal squamous cell cancer. BMC Cancer 8: 98, 2008.

46. Tomasini MD, Wang Y, Karamafrooz A, Li G, Beuming T, Gao J, Taylor SS, Veglia G and Simon SM: Conformational LANDSCAPE of the PRKACA-DNAJB1 chimeric kinase, the driver for fibrolamellar hepatocellular carcinoma. Sci Rep 8: 720, 2018.

47. Petitjean A, Mathe E, Kato S, Ishioka C, Tavtigian SV, Hainaut $P$ and Olivier M: Impact of mutant p53 functional properties on TP53 mutation patterns and tumor phenotype: Lessons from recent developments in the IARC TP53 database. Hum Mutat 28 : 622-629, 2007

48. Olivier M, Hollstein M and Hainaut P: TP53 mutations in human cancers: Origins, consequences, and clinical use. Cold Spring Harb Perspect Biol 2: a001008, 2010.

49. Lawrence MS, Stojanov P, Polak P, Kryukov GV, Cibulskis K, Sivachenko A, Carter SL, Stewart C, Mermel CH, Roberts SA, et al: Mutational heterogeneity in cancer and the search for new cancer-associated genes. Nature 499: 214-218, 2013.
50. Xu L, Hazard FK, Zmoos AF, Jahchan N, Chaib H, Garfin PM, Rangaswami A, Snyder MP and Sage J: Genomic analysis of fibrolamellar hepatocellular carcinoma. Hum Mol Genet 24: 50-63, 2015.

51. Finlay CA, Hinds PW and Levine AJ: The p53 proto-oncogene can act as a suppressor of transformation. Cell 57: 1083-1093, 1989.

52. Harper JW, Adami GR, Wei N, Keyomarsi K and Elledge SJ: The p21 Cdk-interacting protein Cip1 is a potent inhibitor of G1 cyclin-dependent kinases. Cell 75: 805-816, 1993.

53. Kastenhuber ER and Lowe SW: Putting p53 in context. Cell 170: 1062-1078, 2017.

54. Mills KD: Tumor suppression: Putting p53 in context. Cell Cycle 12: 3461-3462, 2013

55. O'Brate A and Giannakakou P: The importance of p53 location: Nuclear or cytoplasmic zip code? Drug Resist Updat 6: 313-322, 2003.

56. Sembritzki O, Hagel C, Lamszus K, Deppert W and Bohn W: Cytoplasmic localization of wild-type p53 in glioblastomas correlates with expression of vimentin and glial fibrillary acidic protein. Neuro Oncol 4: 171-178, 2002.

57. Green DR and Kroemer G: Cytoplasmic functions of the tumour suppressor p53. Nature 458: 1127-1130, 2009.

58. Moll UM, Riou G and Levine AJ: Two distinct mechanisms alter p53 in breast cancer: Mutation and nuclear exclusion. Proc Natl Acad Sci USA 89: 7262-7266, 1992.

59. Bosari S, Viale G, Roncalli M, Graziani D, Borsani G, Lee AK and Coggi G: p53 gene mutations, p53 protein accumulation and compartmentalization in colorectal adenocarcinoma. Am J Pathol 147: 790-798, 1995.

60. Vici P, Di Benedetto A, Ercolani C, Pizzuti L, Di Lauro L, Sergi D, Sperati F, Terrenato I, Dattilo R, Botti C, et al: Predictive significance of DNA damage and repair biomarkers in triple-negative breast cancer patients treated with neoadjuvant chemotherapy: An exploratory analysis. Oncotarget 6: 42773-42780, 2015.

61. Shen X, Zuo X, Zhang W, Bai Y, Qin X and Hou N: MiR-370 promotes apoptosis in colon cancer by directly targeting MDM4. Oncol Lett 15: 1673-1679, 2018.

62. Han H, Wang L, Xu J and Wang A: miR-128 induces pancreas cancer cell apoptosis by targeting MDM4. Exp Ther Med 15: 5017-5022, 2018

63. Zache N, Lambert JM, Rökaeus N, Shen J, Hainaut P, Bergman J, Wiman KG and Bykov VJ: Mutant p53 targeting by the low molecular weight compound STIMA-1. Mol Oncol 2: 70-80, 2008.

64. Bykov VJ, Issaeva N, Shilov A, Hultcrantz M, Pugacheva E, Chumakov P, Bergman J, Wiman KG and Selivanova G: Restoration of the tumor suppressor function to mutant p53 by a low-molecular-weight compound. Nat Med 8: 282-288, 2002.

65. Hiraki M, Hwang SY, Cao S, Ramadhar TR, Byun S, Yoon KW, Lee JH, Chu K, Gurkar AU, Kolev V, et al: Small-molecule reactivation of mutant p53 to wild-type-like p53 through the p53-Hsp40 regulatory axis. Chem Biol 22: 1206-1216, 2015.

66. Bossi G and Sacchi A: Restoration of wild-type p53 function in human cancer: Relevance for tumor therapy. Head Neck 29: 272-284, 2007.

67. Stad R, Little NA, Xirodimas DP, Frenk R, van der Eb AJ, Lane DP, Saville MK and Jochemsen AG: Mdmx stabilizes p53 and Mdm2 via two distinct mechanisms. EMBO Rep 2: 1029-1034, 2001

68. Garcia D, Warr MR, Martins CP, Brown Swigart L, Passegué E and Evan GI: Validation of MdmX as a therapeutic target for reactivating p53 in tumors. Genes Dev 25: 1746-1757, 2011

69. Danovi D, Meulmeester E, Pasini D, Migliorini D, Capra M, Frenk R, de Graaf P, Francoz S, Gasparini P, Gobbi A, et al: Amplification of Mdmx (or Mdm4) directly contributes to tumor formation by inhibiting p53 tumor suppressor activity. Mol Cell Biol 24: 5835-5843, 2004

70. Wang H, Ma X, Ren S, Buolamwini JK and Yan C: A small-molecule inhibitor of MDMX activates p53 and induces apoptosis. Mol Cancer Ther 10: 69-79, 2011.

71. Reed D, Shen Y, Shelat AA, Arnold LA, Ferreira AM, Zhu F, Mills N, Smithson DC, Regni CA, Bashford D, et al: Identification and characterization of the first small molecule inhibitor of MDMX. J Biol Chem 285: 10786-10796, 2010. 